\title{
DEVELOPMENT OF AN R-CUBE BASED GENERAL PURPOSE HAPTIC DEVICE SYSTEM
}

\author{
Tunç Bilgincan \\ Robotics Laboratory \\ Department of Mechanical Engineering \\ İzmir Institute of Technology \\ Urla, İzmir, Turkey 35430 \\ tuncbilgincan@iyte.edu.tr
}

\author{
Mehmet İsmet Can Dede \\ Robotics Laboratory \\ Department of Mechanical Engineering \\ İzmir Institute of Technology \\ Urla, İzmir, Turkey 35430 \\ candede@iyte.edu.tr
}

\begin{abstract}
A novel 6-Degree-of-Freedom (DoF) hybrid haptic device is presented in this paper. Hybrid mechanism consists of parallel kinematic structure, R-CUBE and a 3-DoF orientation mechanism. In our previous study, the original configuration of the R-CUBE mechanism was investigated. In this work, the original design of R-CUBE is reconfigured as a haptic mechanism and the final mechanism is reoriented in order to equally distribute the gravitational effects to all grounded actuators. Rotational motions of the end-effector are monitored through the 3-DoF orientation mechanism placed on the moving platform of the parallel platform. The design of this haptic device is suitable to reflect forces in translational motions, thus point-type of contact is available for this system. The designed device is manufactured utilizing various types of manufacturing processes, such as wire erosion, laser cutting, milling and turning. Finally manufactured mechanism is integrated with electromechanical components and tested for manipulability.
\end{abstract}

\section{INTRODUCTION}

Nowadays, haptics finds use in a wide variety of applications both for scientific and commercial purposes. Robotic surgery [20], robotic rehabilitation [21], nanomanipulation tasks [22], 3D design [23], education and training applications [24] can be listed as some examples where haptics is employed. In general, haptic devices are utilized either in bilateral force-reflecting teleoperation tasks or virtual reality (VR) applications.

Haptic devices are used to increase the telepresence level of the teleoperation. The operator receives more information from the slave side as forces exerted on the environment are transmitted along with the visual information from the slave side of teleoperation. Therefore, sense of touch provided by a haptic device increases the telemanipulability and improves quality of teleoperation. As a result of this, more complex tasks such as controlling a manipulator in hazardous environments or minimal invasive surgery, nano manipulation applications find a solution by making use of haptics in teleoperation settings.

Teleoperation of the underwater construction machines is a typical example of hazardous environment operation.
Hirabayashi et al. [14] employed haptics technology in teleoperation of underwater construction machines for safer and more efficient underwater construction work. Nanomanipulation with haptics was investigated by Sieber et al. [16]. A platform for real-time bilateral biomanipulation was designed and experiments with a cell palpation application were conducted in their work.

Haptic technology increases the level of interaction between the user and the virtual environment in VR applications. Programmed virtual sensors provide sense of touch, which improves the quality of the telepresence. Other than the game industry [25], haptics in VR applications are used frequently in training and educational purpose technologies such as medical simulators, especially for expensive and difficult trainings such as dental trainings. A dental surgery training system with a 6-DoF haptic device [15] is an example for training systems that utilize haptic technology. The device has parallel platform architecture with $100 \times 100 \times 50 \mathrm{~mm}$ of workspace.

Several types of robot arm mechanisms have already been configured as haptic devices. Some of them are designed to have serial kinematic structure $[13,26]$. Their use is limited in applications that require relatively more precision due to their low stiffness, limited force feedback capacity and accumulating joint errors. Although parallel platform mechanisms have limited workspace with respect to serial manipulators, they offer higher stiffness, precision and load capacity ratings because of their structural advantages.

This paper outlines the study towards developing a novel 6DoF hybrid haptic device that is composed of R-CUBE based active 3-DoF translational motion and passive 3-DoF orientation mechanism for rotational motion monitoring. Due to the kinematic advantages of the parallel manipulators, the system is designed to have high stiffness, robustness, precision and high load capacity. The next section describes the previous work on parallel platform based haptic devices. Following the background information, the mechanism design is described. The analyses of the mechanism are later given for forwardinverse kinematics and static force application. Finally, the integration of the mechanical, electromechanical and computer interface is explained and concluding remarks are provided. 


\section{PREVIOUS RESEARCH ON PARALLEL PLATFORM HAPTIC DEVICES}

Various researchers have recently published their work towards developing parallel platform haptic devices. A general purpose 6-DoF haptic interface is proposed in [2]. The structure is a parallel mechanism composed of three serial kinematic chains, each chain having a composition of two active joints and one passive joint. The mechanism is intended to be used as a remote surgical tool. Sabater et al. [3] studied the mechanical design, the geometric and dynamic model, and control algorithms of a 6 URS (Universal-Revolute-Spherical) platform haptic device. Cable-driven pantographs and rotational electric actuators are used to improve the workspace and output bandwidth of the device. A 7-DoF haptic device called PATHOS is developed as a result of the work in [4]. 6-DoF of the device is reserved for the spatial manipulation and the remaining 1DoF for grasping the objects. The parallel mechanism design was chosen to enhance the stiffness and accuracy. Their experimental results indicated that the mechanism can display virtual environment with some stiffness and accuracy.

A 3-DoF wire-based parallel system that provides planar forces and moment feedback is described in [5]. The system is suitable for planar manipulation. Ryu et al. [6] proposed 6-DoF haptic mechanism, which is a combination of a 3-DoF planar lower parallel mechanism and another 3-DoF mechanism mounted on top of the lower mechanism. In order to decrease inertial effects, and to improve backdrivability and transparency, they mounted all the actuators on the ground and actuated all joints with the use of a cable driven system. Lee et al. [7] also presented their work on the design of a 6-DoF parallel haptic device. In their design, they aimed for a light weight system with grounded motors. They performed kinematics analysis and kinematics optimization for their system. In this work, the kinematic optimal design problem is formulated and Genetic Algorithm method is utilized to find an optimal solution. Gosselin et al. [8] developed a new 6-DoF haptic device for desktop applications. Their device is a lightweight parallel-structured mechanism with the handle on its moving platform.

Frisoli et al. [9] introduced a two point contact, portable haptic interface for the thumb and index fingers of the hand. Optimization techniques are used for determining the kinematic dimensions of their device. Yoon et al. [10] designed a novel 6DoF haptic mechanism that is driven with servomotors. The orientation mechanism is also placed on the moving platform of the parallel mechanism in this study. Kwon et al. [11] presented a 2-DoF haptic device equipped with electrical motors and brakes. Every active joint is actuated by a pair of a motor and a brake. They tested the system for modeled environments, such as the contact with a virtual wall and frictional effects due to this contact. A new spherical mechanism for a haptic system called "Shade" is described in [12]. Due to the spherical geometry, the mechanism has pure rotation around it center point, where the handle of the joystick is located at.

Investigating the previous work on developing new parallel platform haptic devices, design specifications for our system are determined. Design criteria and procedure is outlined in the next section.

\section{DESIGN OF THE MECHANISM}

The haptic device is designated to reflect only the threedimensional forces. Point type of contact can be simulated as a result of this configuration. Therefore, in VR applications, only point type of contact can be modeled, and in teleoperation applications, only the measured slave forces but not the moments can be fed-back. Nevertheless, it is a requirement to provide control signals in 6-DoF to control spatial systems. Design criteria of the mechanism design are listed in Table 1.

\section{Table 1. DESIGN CRITERIA OF THE HAPTIC DEVICE}

\begin{tabular}{|l|l|}
\hline Footprint & $\sim 210 \mathrm{~mm}^{2}$ \\
\hline Force feedback workspace & $120 \mathrm{~W}$ x $120 \mathrm{H}$ x 120 D mm \\
\hline Force feedback & $\vec{u}_{1}, \vec{u}_{2}, \vec{u}_{3}$ \\
\hline Continuous exertable force & $>0.8 \mathrm{~N}$ \\
\hline Nominal position resolution & $0.03 \mathrm{~mm}$ \\
\hline
\end{tabular}

Evaluating the basic requirements outlined above, a hybrid formation is selected to uncouple the translational and rotational motions. Parallel platform mechanisms for translational motion and force reflection and mechanisms for rotational motion detection are investigated. Thus, possible hybrid structures are studied, conceptually designed and analyzed for their functionality, ease of control and production, and cost. The mechanisms investigated for translational motions are Cartesian Parallel Platform, Delta and R-CUBE, whereas Agile Eye, Hybrid Spherical Manipulator and Serial Spherical Manipulator are the mechanisms investigated for rotational motions. The conceptual designs and their analyses are detailed in [17]. RCUBE and Serial Spherical Manipulator mechanisms are selected as they met the specifications for having all revolute joints, grounded actuators, and minimum amount of links and joints. CAD (Computer-Aided-Design) drawing of the final design for the full mechanism with its actuators and sensors is shown in Fig. 1.

The translation and orientation mechanism is described in the following subsections. The structural and electromechanical parts, such as actuators and sensors, are described with their locations on the mechanism. In the last subsection, the manufacturing process of the structural parts of the mechanism is detailed.

\section{Translation Mechanism}

R-CUBE manipulator is a 3DoF-3NRRR parallel manipulator that can generate only translational motions having 
only revolute joints. It has three kinematic serial chains attached to the moving platform with four links on each chain. The mechanism has actuators placed on the ground. The number of joints is twenty-one and the number of links is seventeen including base and platform of the manipulator. The motion of the manipulator is de-coupled for each translational motion. Thus, the kinematics analysis of the manipulator is relatively simple.

The motion and the workspace limits are directly related with the length and the position of the first link of the manipulator that is denoted with " 1 " in Fig. 1. The singularities of the manipulator exist only at the limits of the workspace, which is not possible to reach for this configuration [1].

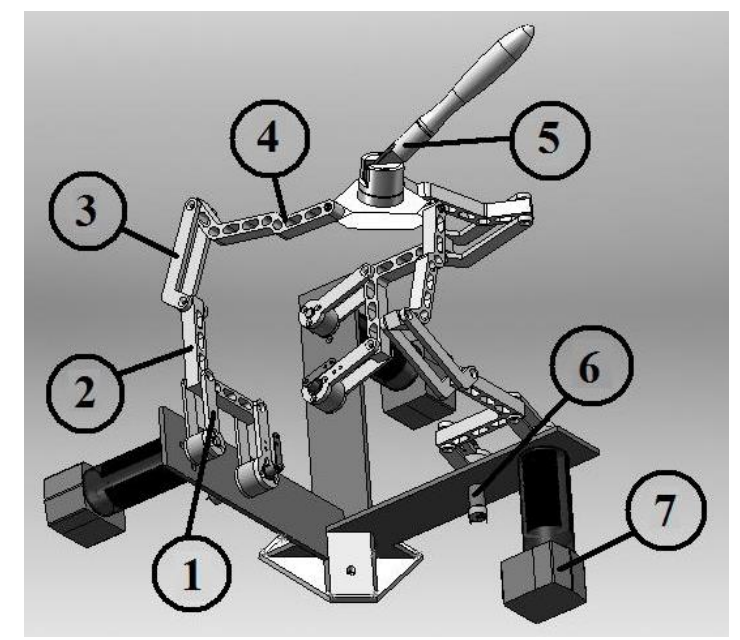

Figure 1. CAD DRAWING OF 6-DoF MECHANISM.

R-CUBE structure has unique properties to be configured as a haptic device mechanism considering the properties outlined above. The structure of the mechanism with all revolute joints calls for rather simpler manufacturing methods; thus decreases the cost. The decoupled motion results in straightforward forward kinematics solution and static force analysis of the system. In haptics, due to the random motion of the operator, motion through singular positions must be restricted. Singularity analysis of the system is discussed in [1] and R-CUBE allows prevention of the motion through singularities with proper design.

The design process of the mechanism in this work is carried out in two main work packages; (1) Modification of the mechanism complying with the test result from the initial test mechanism [17], (2) Definition of the mechanism parameters, such as link lengths to avoid singularity and collusions of the links. The original structure is reoriented in order to equally distribute the gravitational effects to all grounded actuators. Length of the fist links is calculated by forward kinematics analysis formula presented in Eqn. (2) and (3) to meet the workspace limitation specification. The location of the workspace is defined by $S$ parameter in Eqn. (2) and (3) based on the ergonomic reasons. The lengths of the second link denoted as " 2 ", the third link as " 3 ", and the fourth link as " 4 " in Fig. 1 are determined to avoid possible singularities and link collusions. The link length determination process is based on the mechanism simulation carried out in SolidWorks@ CosmosMotion ( ) (a CAD and mechanism analysis software). Handling space that is required for operator's hand to function without any collusion is another design parameter of the mechanism. The ergonomics of the handle is also evaluated in the CAD design phase.

The aim of this work is to design high precision and compact mechanism. Hence, bearings and rigid material is selected to be utilized in the design. The precise sensor and actuator systems used to meet the high precision requirements are denoted as " 6 " and " 7 " respectively in Fig. 1 . The parallel platform structure of the mechanism enables to integrate different types of end-effector and orientation mechanisms on top of the moving platform. The current selection of the orientation mechanism and the end-effector is indicated with " 5 " in Fig. 5. The next subsection provides more information on the orientation mechanism.

\section{Orientation Mechanism}

The orientation mechanism is a 3-DoF-RRR spatial serial mechanism. The structure of the orientation mechanism has the spherical wrist composition of an industrial arm. The rotation axes of the joints intersect at the wrist point, $P$, as shown in Fig. 2. The forward kinematics analysis of both translation and orientation mechanisms are carried out in the next section.

The primary challenge in the design of this mechanism is adjusting the size of the mechanism with large amount of components, such as position sensors (trimpot), cables and A/D converters. Nevertheless, data acquisition components, sensors and bearings were concealed into the mechanism in consideration of the handle ergonomics. The locations of the sensors and the rotation axes of the joints are shown in Fig. 2.

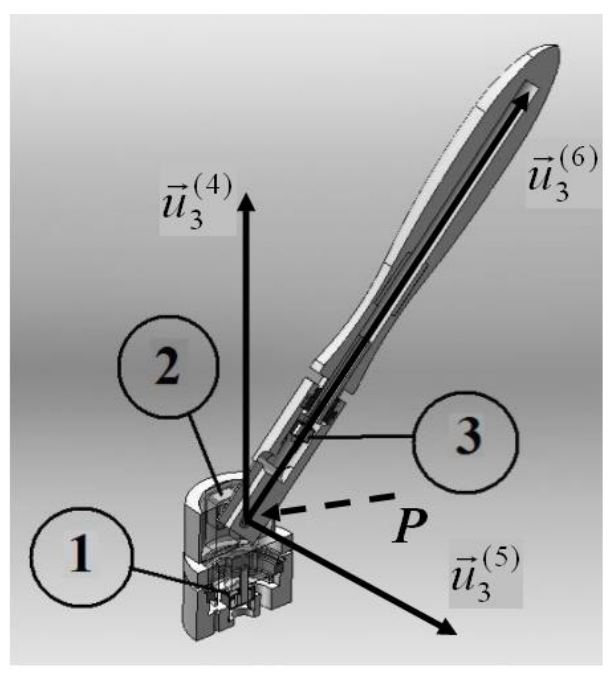




\section{Figure 2. SENSOR LOCATIONS AND ROTATION AXES OF THE ORIENTATION MECHANISM.}

The end-effector is designed to have a pen-like shape, but any type of end-effector can be attached to the orientation mechanism as required by the specific task. The orientation mechanism is also fully detachable and a rigid end-effector can be placed if the task does not require collecting orientation data from the operator.

\section{Manufacturing Process}

The designed device is manufactured utilizing various types of manufacturing process, such as wire erosion, laser cutting, milling and turning for translational mechanism. The manufacturing process was challenging due to the use of aluminum alloy (7075), which is light-weight, non-corrosive, and durable, and has good fatigue strength. This material is used to manufacture the links. Another challenging issue is the manufacturing of bearing assemblies for small-scale bearings to fit in the compact design. Bearing assemblies and the links are produced by the wire corrosion technique in order to satisfy the precision requirements.

The base of the translational mechanism was manufactured by laser cutting technology from stainless steel. The base carries the actuators and the mechanism at all times, thus the high strength material, stainless steel, is selected as the material of this part. Shafts of the small scale bearings are manufactured by CNC milling from brass for its strength, abrasion resistance and its endurance for corrosion. Potentiometer housings of the translation mechanism were also manufactured by the same milling process.

Moving platform of the mechanism was produced from the polyoxymethylene plastic, commonly known as Delrin material, by turning operation. It was also a challenging operation to manufacture the moving platform of the mechanism due to its distinct shape. The orientation mechanism is also produced from another plastic material, Kestamid $\odot$ (cast polyamide). Plastic materials are used to manufacture the extremities of the mechanism in order to reduce the total payload amount. Finally, all links are perforated in order to decrease the weight of the links.

\section{KINEMATICS AND STATIC FORCE ANALYSES}

In the parallel mechanism (R-CUBE), the motion of the platform is controlled by the rotation of the actuators. Due to the decoupled motion of the mechanism, the motion along $\vec{u}_{1}$, $\vec{u}_{2}$, and $\vec{u}_{3}$, axes is dependent only on the motors which have rotations about $\vec{u}_{2}, \vec{u}_{3}$, and $\vec{u}_{1}$ axes respectively. Therefore, the mechanism's forward and inverse kinematics analysis is relatively trivial. The mechanism parameters and the ground coordinate frame are presented in Fig. 3. In the forward and inverse kinematics analysis, and in the static torque calculations, the notation in Fig. 3 is used. The wrist point position, $P$, is defined as the intersection point of the last link connection surfaces on the moving platform. The main coordinate frame origin point, $O$, is located at the intersection point of unit vectors of the coordinate frame that originate from the respective actuator axes in Fig. 3.

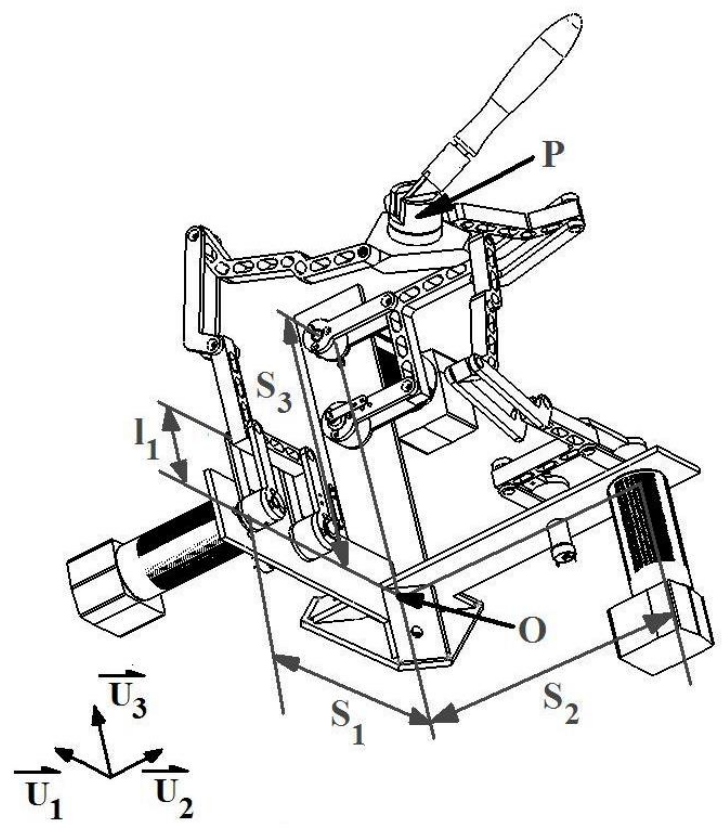

Figure 3. MECHANISM PARAMETERS.

The torque and position analysis of the system is carried out in rotated coordinate frame as shown on the bottom left corner of Fig. 3. Nevertheless, for VR or teleoperation applications coordinate frame is reoriented with rotation sequence [18] presented in Eqn. (1). The reorientation angles, $\alpha$, and $\beta$, are calculated using the isometric projection representation shown in Fig. 4.

$$
\begin{gathered}
\hat{C}^{(R, W)}=e^{\tilde{u}_{1} \alpha} e^{\tilde{u}_{2} \beta} \\
\alpha=\arcsin \left(\tan 30^{\circ}\right) \cong 35.26^{\circ} \\
\beta=-45^{\circ}
\end{gathered}
$$

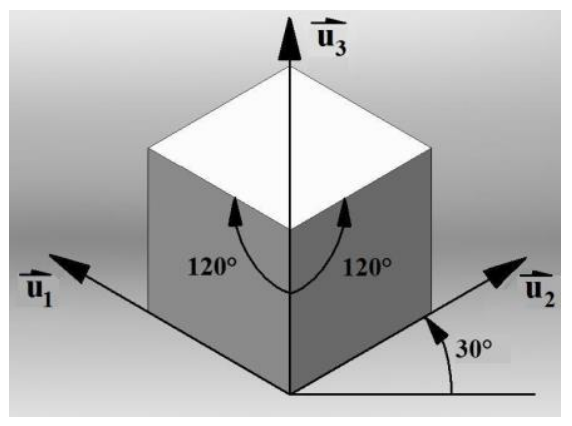

Figure 4. ISOMETRIC PROJECTION 
Eqn. (2) describes position vector of the wrist point, $P$, with respect to the origin point, $O$, in the ground reference frame presented in Fig. 3.

$$
\vec{P}=P_{1} \vec{u}_{1}+P_{2} \vec{u}_{2}+P_{3} \vec{u}_{3}
$$

Components of the wrist point vector, $\vec{P}$, are calculated as a function of the rotation of the actuators, $\theta_{i}$, as shown in Eqn. (3). The rotation angle, $\theta_{i}$, is selected to be zero at the position where the first link is perpendicular to the respective axis as shown in Fig. 5. In Eqn. (3), $S_{i}$ defines the distance between motor axes and origin point along the respective axis, $\vec{u}_{i}$, and $l_{i}$ is the effective link length of the first link.

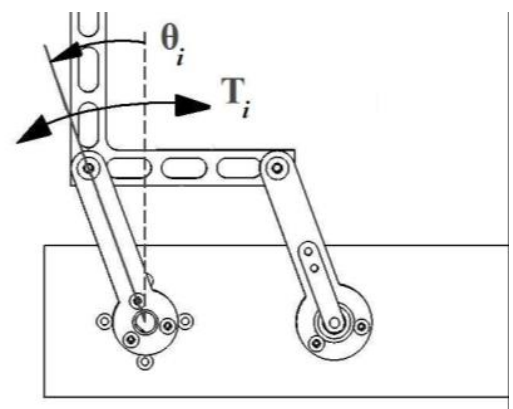

Figure 5. JOINT VARIABLE $\left(\theta_{i}\right)$ AND TORQUE $\left(T_{i}\right)$ DIRECTIONS

$$
P_{i}=S_{i}+l_{i} \sin \left(\theta_{i}\right) ; \quad i=1,2,3
$$

The inverse kinematics solution is originated from Eqn.3 and represented by the set of equations given in Eqn. (4).

$$
\begin{gathered}
\sin \left(\theta_{i}\right)=\frac{P_{i}-S_{i}}{l_{i}} \quad i=1,2,3 \\
\cos \left(\theta_{i}\right)=\sigma \sqrt{1-\sin ^{2}\left(\theta_{i}\right)} ; \quad \sigma= \pm 1 \\
\theta_{i}=\arctan _{2}\left(\sin \left(\theta_{i}\right), \cos \left(\theta_{i}\right)\right)
\end{gathered}
$$

The mechanical structure of the four-bar mechanism limits the motion of the first link to $\pm 65^{\circ}$. Therefore, it is not possible to pass to the region that the cosines of the joint variables become negative. As a result of this, the sign ambiguity denoted with $\sigma$ in Eqn. (4) can be neglected and $\sigma$ can be taken as +1 without the loss of generality.

The orientation mechanism is in the form of a spherical wrist configuration. Thus, its forward kinematics analysis is provided as a series of pure rotations in Eqn. (5). The orientation mechanism is a passive mechanism. The inverse kinematics solution is not required since the motion of the mechanism is not controlled by any actuation system.

$$
\hat{C}^{(3,6)}=e^{\tilde{u}_{3} \theta_{4}} e^{\tilde{u}_{2} \theta_{5}} e^{\tilde{u}_{3} \theta_{6}}
$$

Static force analysis is essential in haptics since the primary objective is to restrain the operator's motion with calculated forces. The components of the applied force, $F$, are calculated in the ground reference frame in Eqn. 6.

$$
\vec{F}=F_{1} \vec{u}_{1}+F_{2} \vec{u}_{2}+F_{3} \vec{u}_{3}
$$

Torque demands for a specified force that is required to be applied to the operator's hand can be formulated by Eqn. (7). In Eqn. (7), $T$ is the torque required to be applied by the actuators about their respective axis of rotation as shown in Fig. 5 , and $F$ is the desired force to be applied to the operator's hand. Torque values for each actuator are calculated by taking cross product of the moment arm vector, $\vec{P}$, and the force vector, $\vec{F}$, and then taking the dot product with the respective actuator's rotation axis. The torque element in all axes that has the moment arm along its respective axis is neglected because the forces will not be transferred as a consequence of the rotation axis arrangement of links 2, 3, and 4 .

$$
\begin{aligned}
& T_{1}=F_{1}\left(P_{3}\right) \\
& T_{2}=F_{2}\left(P_{1}\right) \\
& T_{3}=F_{3}\left(P_{2}\right)
\end{aligned}
$$

\section{INTEGRATION OF THE SYSTEM}

The system is composed of mechanical parts, electromechanical and electronics components. The mechanism is manufactured and assembled as described in the previous section and shown in Fig. 5 with "2". Actuators and sensors were required to be selected to be integrated to the system as well as the electronics that drive and monitor these electromechanical components. The components are described in the order of actuators and their drivers, motion sensors, and data acquisition system.

The actuators are selected as a result of the force analysis performed in the CAD simulations. The applied force specification is to apply at least $0.8 \mathrm{~N}$ of force in all directions. This specification is consistent with the similar haptic devices in the market [13]. The initial selection of the actuator is a Maxon brushless DC motor (BLDC) that provides $310 \mathrm{mNm}$ of torque at $5250 \mathrm{rpm}$, which is denoted as " 5 " in Fig. 5. The relatively high torque is required since no transmission system is used between the actuators and the mechanism. In haptic devices, usually DC motors with capstan drives are used. However, it is foreseen that in time, the cables of the capstan drives loosen and results in loss of precision. Maxon motor amplifiers (driver) are 
used to drive the actuators in current mode, which enables to drive the actuators by torque inputs that originated from the haptic controller system. The drivers are selected to have a response rate of at least $1 \mathrm{kHz}$. The drivers are indicated with "4" in Fig. 5.

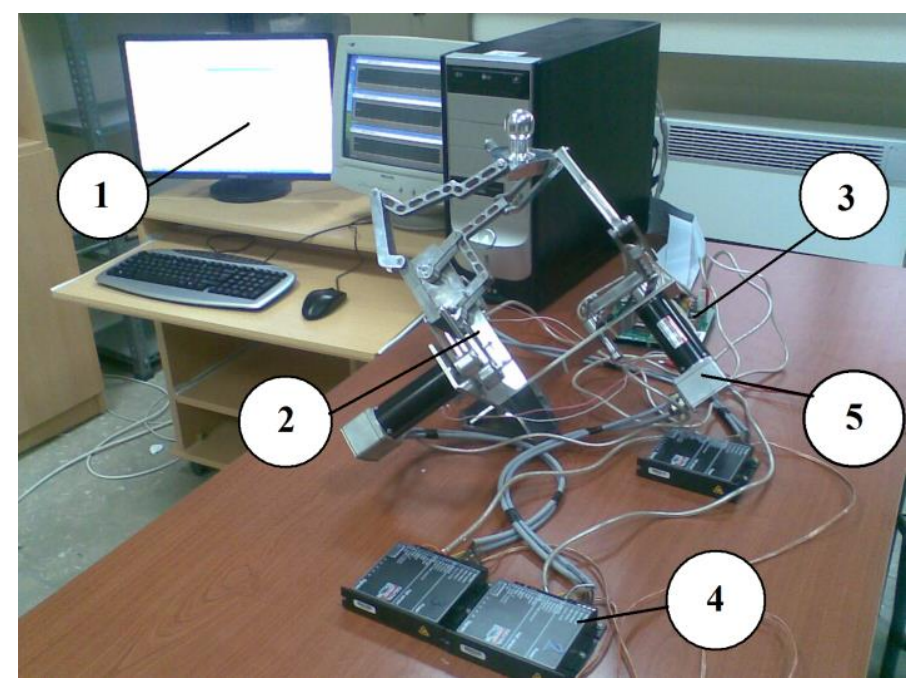

Figure 5. INTEGRATED HAPTIC DEVICE SYSTEM.

The configuration of the mechanism allows using a separate sensor to measure the rotation amount of each actuator. Thus, it is not required to have an encoder system at the rear end of the actuator which usually has 9-bit of resolution. The four-bar structure placed as the fist link has identical nominal lengths, which permits the same motion on each link. A potentiometer or a higher bit resolution encoder (12-bit) can be aligned with the free link of the four-bar system and measure the same motion with more precision. Initially, a potentiometer system is selected to measure the motion.

While human motion is limited to $10 \mathrm{~Hz}$, forces that are up to $1 \mathrm{kHz}$ is important for the perception of the outer media. Therefore, in general, haptic devices are required to have at least $1 \mathrm{kHz}$ of sampling rate in order to accurately provide the sense of touch [19]. Haptic device has to send and receive data through a protocol to be integrated with the computer and the haptic control system. A data acquisition (DAQ) card is used to convert analogue inputs from the potentiometers to digital signals to be fed into the computer and digital outputs from the computer to analogue signals to be fed into the motor drivers.

The electromechanical components of the system require exchanging data with the computer where the haptic control algorithm will be running. Six potentiometers that provide analogue signals, three on the parallel mechanism - three on the orientation mechanism, are used in the system. Another system that requires a computer interface is the motor driver systems that drive the three BLDC motors in current mode requiring +/$10 \mathrm{~V}$ of analogue signals as inputs. The exchange of these signals with the computer has to be at a rate of $1 \mathrm{kHz}$. The Q8
Hardware in the Loop (HIL) Board from Quanser Consulting Inc. is selected to meet the requirements. Q8 has eight 12-bit D/A converters, eight 14-bit A/D converters, eight quadrature encoder inputs and thirty-two digital I/O channels. The terminal board of Q8 is denoted as " 3 " in Fig. 5. The system currently occupies three D/A converters for motor drivers and six A/D converters for the potentiometers of the Q8.

In a VR application, acquired signals from the motion sensors through the DAQ card are used to move a virtual object in VR screen. The forces calculated as a result of the interaction between the virtual object and the virtual environment is then sent to the mechanism through the DAQ card. Figure 6 summarizes the data flow of the integrated system.

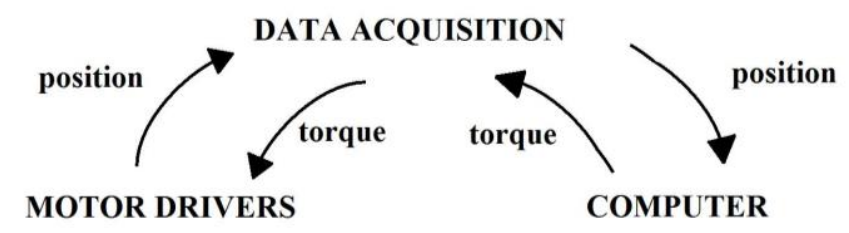

MECHANISM

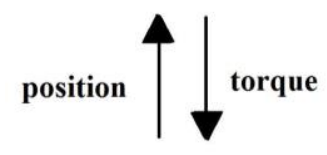

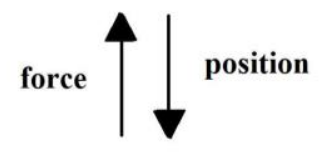

VIRTUAL REALITY/ TELEOPERATION
Figure 6. DATA FLOW

The only difference in teleoperation integration of this haptic device would be that actual forces has to be measured on the slave side of teleoperation instead of calculating virtual forces in the VR environment. The slave system is usually at a distant site. Therefore, the signals between the master and the slave robot must have a communication system such as the Internet, cables or wireless technology in teleoperation systems.

\section{CONCLUSIONS}

In this work, the aim was to develop a haptic device that has higher precision ratings. Parallel platform mechanisms that were configured as haptic devices are investigated as they have higher payload capacities along with the higher precision specifications. After evaluating the previous designs and our conceptual designs, R-CUBE mechanism is selected for reflecting three-dimensional forces and $3 R$ serial spatial mechanism is selected for monitoring the orientation of the operator's hand. Thus, a novel hybrid mechanism is configured. The R-CUBE mechanism with its original configuration has been built and tested previously by the authors in their previous [17]. As a result of this evaluation, the mechanism is reoriented to distribute the payload evenly between the actuators.

The mechanism is analyzed and mechanism parts are designed to meet the design criteria. Following the analyses, the 
mechanism is manufactured. The mechanism is integrated using the produced mechanism, selected BLDC motors and their amplifiers, the potentiometers, the DAQ equipment and the computer. The authors are currently conducting gravity cancellation tests with the integrated system.

The performance of the device will be evaluated with also brushed DC motors and encoders replacing the BLDC and potentiometers for future investigations.

\section{ACKNOWLEDGMENTS}

This research was supported by a Marie Curie International Reintegration Grant within the $7^{\text {th }}$ European Community Framework Programme.

\section{REFERENCES}

[1] Li, W., Gao, F., Zhang, J., 2005. "R-CUBE a Decoupled Parallel Manipulator Only with Revolute Joints". Mechanism and Machine Theory, 40(4), April, pp. 467473.

[2] Lee S, S., Lee, J, M., 2003. "Design of a General Purpose 6-DoF Haptic Interface”, Mechatronics, 13, pp. 697-722.

[3] Sabater, J.M., Saltarén, R.J., Aracil, R., 2004. "Design, Modelling and Implementation of a 6 URS Parallel Haptic Device". Robotics and Autonomous Systems, 13(7), September, pp. 1-10.

[4] Kim, K., Chung, W. K., 2003. "Design and Analysis of a New 7-DoF Parallel Type Haptic Device: PATHOS-II". Proceedings of the 2003 IEEE Conference on Intelligent Robots and Systems, Vol. 3, IEEE, ,pp. 2241-2246.

[5] Gallina P., Rosati, G., 2007. "Manipulability of a Planar Wire Driven Haptic Device". Mechanism and Machine Theory, 37(2), February, pp. 215-228.

[6] Ryu D., Song, J., Cho, C., Kang, S., Kim, M., 2010. "Development of a Six DoF Haptic Master for Teleoperation of a Mobile Manipulator". Mechatronics, Elsevier, 20(2), March, pp. 181-191.

[7] Lee, J. H., Eom, K. S., Yi, B-J., Suh I. H., 2001. "Design Of A New 6-DoF Parallel Haptic Device". Proceedings of the 2001 IEEE International Conference on Robotics \& Automation, Vol. 1, IEEE, pp. 886-891.

[8] Gosselin, F., Martins, J., Bidard, C., Andriot, C., Brisset, J., 2005. "Design of a New Parallel Haptic Device for Desktop Applications". Proceedings of the First Joint Eurohaptics Conference and Symposium on Haptic Interfaces for Virtual Environment and Teleoperator Systems, IEEE, pp. 295-299.

[9] Frisoli, A., Simoncini, F., Bergamasco, M., Salsedo, F., 2007. "Kinematic Design of a Two Contact Points Haptic Interface for the Thumb and Index Fingers of the Hand". Journal of Mechanical Design, 129(5), May, pp. 520-529.

[10] Yoon, J., Ryu, J., Design, 2001. "Fabrication and Evaluation of a New Haptic Device Using a Parallel
Mechanism". Transactions on Mechatronics, 6(3), September, pp. 221-233.

[11] Kwon, T., Song, J., 2006. "Force Display Using a Hybrid Haptic Device Composed of Motors and Brakes". Mechatronics, 16(5), June, pp. 249-257.

[12] Birglen, L., Gosselin, C., Pouliot, N., Monsarrat, B., Laliberté, T., 2002. "SHaDe, a New 3-DoF Haptic Device". IEEE Transactions on Robotics and Automation, 18(2), April, pp. 166-175.

[13] Massie, T.H., Salisbury, J.K. 1994. "The PHANTOM haptic interface: a device for probing virtual objects". Proceedings of the ASME Winter Annual Meeting, Symposium on Haptic Interfaces for Virtual Environment and Teleoperator Systems, Vol. 55-1, ASME, pp.263-267.

[14] Hirabayashi, T., Akizono, J., Yamamoto, T., Sakai, H., Yano, H., 2006. "Teleoperation of Construction Machines with Haptic Information for Underwater Applications". Automation in Construction, 15(5), pp. 563 - 570.

[15] Cao, Y., Zhang, Y., 2007. Member, Yunzhong Ma, and Dangxiao Wang, "Design and Analysis of a New Six-DoF Haptic Device for Dental Training". 16th IEEE International Conference on Robot \& Human Interactive Communication, IEEE, July, pp.263-267.

[16] Sieber, A., Valdastri, P., Houston, K., Eder, C., Tonet, O., Menciassi, A., Dario, P., 2008. "A Novel Haptic Platform for Real time Bilateral Biomanipulation with a MEMS Sensor for Triaxial Force Feedback". Sensors and Actuators, 142(1), March, pp. 19-27.

[17] Dede, M. I. C., Selvi, Ö., Bilgincan, T., Kant, Y., 2009. "Design of a Haptic Device for Teleoperation and Virtual Reality Systems". Proceedings of the IEEE International Conference on Systems, Man \& Cybernetics, IEEE, pp. $3623-3628$.

[18] Carlbom, I., 1978. "Planar Geometric Projections and Viewing Transformations". Computing Surveys, 10(4), December, pp. 465-502.

[19] Tanner, N. A., Niemeyer, G., 2006. "High-Frequency Acceleration Feedback in Wave Variable Telerobotics". IEE/ASME Transactions on Mechatronics, Vol. 11, IEE/ASME, pp. 119-127.

[20] Katsura, S., Iida, W., Ohnishi, K., 2005. “An Application to Haptic Forceps". Medical Mechatronics, 29(2), November, pp. 237-245.

[21] Schmidt, H., 2004. "HapticWalker - A Novel Haptic Device for Walking Simulation". Proceedings of EuroHaptics 2004, Vol. 2, EuroHaptics , pp. 60-67.

[22] Wollacott, A.M., Merz Jr, K.M., 2007. "Haptic Applications for Molecular Structure Manipulation”. Journal of Molecular Graphics and Modelling, 25(6), March, pp. 801-805.

[23] Gao, Z., Gibson, I., 2008. "Haptic Sculpting of MultiResolution B-spline Surfaces with Shaped Tools”. 
Computer-Aided Design Archive, 38(6), June, pp. 10551066.

[24] Wang, P., Becker, A.A., Jones, I.A., Glover, A.T., Benford, S.D., Greenhalgh, C.M., Vloeberghs, M., 2006. "A Virtual Reality Surgery Simulation of Cutting and Retraction in Neurosurgery with Force-feedback". Computer Methods and Programs in Biomedicine, 84(1), October, pp. 11-18.
[25] Park, K., Bae, B., Koo, T., 2004. “A Haptic Device for PC Video Game Application”. Mechatronics, 14(2), March, pp. 227-235.

[26] SensAble Technologies, March 2009. http://www.sensable.com/products-haptic-devices.htm. 